\title{
Characterization of RsgWBDC053: A New Greenbug Resistance Gene From Wild Barley (Hordeum Vulgare ssp. Spontaneum)
}

Xiangyang Xu ( $\sim$ xiangyang.xu@ars.usda.gov )

USDA-ARS https://orcid.org/0000-0002-1364-7941

Dolores Mornhinweg

USDA-ARS Wheat Peanut and Other Field Crops Research Unit

Amy Bernardo

USDA-ARS Hard Red Winter Winter Wheat Research Unit

Genqiao Li

USDA-ARS Wheat Peanut and Other Field Crops Research Unit

Ruolin Bian

Kansas State University

Brian J. Steffenson

University of Minnesota Department of Plant Pathology

Guihua Bai

USDA-ARS Hard Red Winter Wheat Genetic Research Unit

\section{Research Article}

Keywords: Greenbug, Wild Barley

Posted Date: May 17th, 2021

DOl: https://doi.org/10.21203/rs.3.rs-496936/v1

License: (c) (i) This work is licensed under a Creative Commons Attribution 4.0 International License.

Read Full License 


\section{Abstract}

Greenbug (Schizaphis graminum Rondani) is a destructive insect pest that not only damages plants, but also serves as a vector for many viruses. Host plant resistance is the preferred strategy for managing greenbug. To date, only two greenbug resistance genes, Rsg1 and Rsg2, have been reported in barley, with only the former being deployed in cultivars. To breed cultivars with effective resistance against various greenbug biotypes, additional resistance genes are urgently needed to sustain barley production. Wild barley accession WBDC053 (PI 681777), originating from the Baluchistan region of Pakistan, was previously found to be resistant to several greenbug biotypes. In this study, a recombinant inbred line (RIL) population derived from Weskan $\times$ WBDC053 was evaluated for response to greenbug biotype $E$ and genotyped using genotyping by sequencing (GBS). A set of 3,347 high quality GBS-derived single nucleotide polymorphisms (SNPs) were then used to map a greenbug resistance gene in this wild barley accession. Linkage analysis placed the greenbug resistance gene in WBDC053, temporarily designated RsgWBDC053, in a $2.35 \mathrm{Mb}$ interval $(0-2,354,645 \mathrm{bp})$ in the terminal region of the short arm of chromosome $2 \mathrm{H}$. This interval harbors 15 genes with leucine-rich-repeat (LRR) protein domains. An allelism test between WBDC053 carrying RsgWBDC053 and STARS1501B carrying Rsg2 indicated that the former is either allelic or closely linked to the latter. GBS-SNPs $2 H_{-} 1318811$ and $2 H_{-} 1839499$ cosegregated with RsgWBDC053 and were converted to Kompetitive allele specific PCR (KASP) markers, KASP-Rsg053-1 and KASP-Rsg0533-2. The two KASP markers can be used to select for RsgWBDC053, but also have the potential to tag $R s g 2$ in barley improvement programs.

\section{Key Message}

A new gene for greenbug resistance (RsgWBDC053) was identified in the terminal region of chromosome $2 \mathrm{HS}$ in wild barley (Hordeum vulgare ssp. spontaneum), and two KASP markers co-segregating with the gene were developed for use in introgressing greenbug resistance in barley breeding programs.

\section{Introduction}

Barley (Hordeum vulgare L.) is one of the most widely grown crops in the world and is ranked fourth in world production among food crops in 2017 (FAOSTAT, 2017). The crop has a number of important uses, including malt production for beer and spirits, feed for animals and also food for human consumption (Ullrich 2011). Barley is often cultivated in marginal areas with few additional inputs. Thus, it is important to protect the crop from biotic constraints such as insects to secure sustainable and economic production.

Greenbug (Schizaphis graminum Rondani) is one of the most important insect pests in US barley production, with large scale outbreaks occurring every 5-10 years in the southern Great Plains (Starks and Burton 1997; Giles et al. 2000, 2003). Greenbug feeding causes chlorosis, necrosis and even death of plants when they are heavily infested. Although outbreaks occur only occasionally, yield losses can be huge in an outbreak year without expensive pesticide applications. 
Growing greenbug resistant barley is the preferred approach for insect control because it reduces production costs and eliminates contamination from pesticide applications. Although considerable efforts have been made to identify sources of resistance, only two barley greenbug resistance genes, $R s g 1$ and $R s g 2$, have been identified. Rsg 1 was found in barley cultivar 'Post 90', a reselection of barley cultivar 'Post' derived from the cross Harrison $x$ Will with Will as the donor of the resistance gene (Edwards et al. 1985; Mornhinweg et al. 2004). Rsg2 was first identified in Jao (PI 426756), a spring barley landrace collected from Sindh, Pakistan in 1976. Two breeding lines carrying Rsg2, STARS1501B and STARS1502B, were released in the US in 2018 (Mornhinweg et al. 2018). Previous studies indicated that $R s g 2$ was non-allelic to Rsg 1 (Webster and Starks 1984), and that the two genes responded differently to greenbug biotypes TX1, WY10MC, WY81, and WY10B (Porter et al. 2007; Armstrong et al. 2016). Rsg1 was mapped to an 8.4-cM interval in the terminal region of chromosome $3 \mathrm{HL}$ using SSR and SNP markers (Azhaguvel et al. 2014) and has been used in barley breeding. However, Rsg2 has not been used in breeding and its genomic location remains unknown.

$R s g 1$ and Rsg2 confer resistance to some economically important greenbug biotypes. However, new virulent greenbug biotypes continuously occur. For example, Armstrong et al. (2016) identified six new greenbug biotypes in Wyoming alone, including WY81, WY10MC, WY10B, WY12 MC, WY86 and WY4. Among these, biotype WY81 is virulent to Rsg1, while WY10MC and WY10B are virulent to Rsg2. Therefore, new greenbug resistance genes are urgently needed in barley breeding pipelines to manage greenbug.

Wild barley (Hordeum vulgare ssp. spontaneum) is the progenitor of cultivated barley and originated in the Fertile Crescent region. Today, its range extends from North Africa in the west, across Central Asia and into Southern Asia. Wild barley possesses abundant genetic variation and is considered an important source of biotic and abiotic stress resistance. To identify new genes for disease resistance in barley breeding, Steffenson et al. (2007) assembled the Wild Barley Diversity Collection (WBDC), comprised of 318 accessions from the Fertile Crescent, Central Asia, North Africa, and the Caucasus regions. The WBDC was screened for reaction to a set of greenbug biotypes by the USDA-ARS, Stillwater, OK, and only a few accessions were found resistant. One of these accessions (WBDC053) exhibited a unique response profile to a suite of greenbug biotypes, with resistance to greenbug biotypes $B, C, E, I, T X 1$, WY4A, WY4B, WY81, WY12MC, and WY86 and susceptibility to F, H, WY10MC, and WY10B (Armstrong et al. 2016). Therefore, WBDC053 may carry new greenbug resistance gene(s). The objectives of this study were to characterize the genetics of greenbug resistance in WBDC053 and develop genomic tools for its introgression into elite barley cultivars.

\section{Materials And Methods}

\section{Mapping population}

A set of $154 \mathrm{~F}_{5: 6}$ recombinant inbred lines (RILs) was developed from the cross Weskan $\times$ WBDC053 using single seed descent and used in this study. WBDC053 (PI 681777) is a wild barley accession 
originally collected in the Baluchistan region of Pakistan. It was derived from a single plant selection and selfed five times at University of Minnesota, St Paul, MN. (Ames et al. 2015). Weskan, a winter barley cultivar with a pedigree of Purdue 6515A2/KY 66-7-63-1294, was released by the Kansas State University Experiment Station in 1990 and is susceptible to greenbug.

\section{Evaluation of RILs for responses to greenbug biotype $E$}

WBDC053, Weskan and all RILs were evaluated for responses to greenbug biotype $E$ at the USDA-ARS Wheat, Peanut, and Other Field Crop Research Unit, Stillwater, OK in 2020. The experiment was conducted in a greenhouse with supplemental light of $14 \mathrm{~h}$ per day at $22 \pm 5^{\circ} \mathrm{C}$. Greenbug biotype $\mathrm{E}$ was increased on susceptible barley cultivar Eight-Twelve (PI 537437). A randomized complete block design with two replicates was used. Screening flats were $152 \mathrm{~cm}$ long and $104 \mathrm{~cm}$ wide with $8 \mathrm{~cm}$-diameter hills spaced $8 \mathrm{~cm}$ apart in the formation of 60 hills $(6 \times 10)$ per flat. For each RIL, six seeds were planted in each of two consecutive hills in each replicate, and the two parents were planted in each flat as resistant (WBDC053) and susceptible (Weskan) controls. Flats were infested as soon as seedlings emerged by laying heavily infested leaves from culture pots of greenbug biotype $\mathrm{E}$ between the rows. Plants were rated approximately two weeks after infestation when the susceptible checks had died. The dead plants were classified as susceptible, and the normal plants were classified as resistant.

\section{Genotype the RIL population using the genotyping-by-sequencing}

Leaf tissue was collected from each RIL and the two parents at the two-leaf stage, dried at $-80^{\circ} \mathrm{C}$ in a Freezemobile 35EL Sentry 2.0 Lyophilizer (Sp Scientific, Warminster, PA), and grounded at 1,500 rpm for one min in a MiniG® Automated Tissue Homogenizer (Metuchen, NJ, USA). Genomic DNA was extracted using a method described by Dubcovsky et al. (1994) and quantified using the Quant-iT ${ }^{\mathrm{TM}}$ PicoGreen dsDNA assay kits (Thermo Fisher Scientific, Waltham, MA, USA). A protocol described by Mascher (2013) was used to construct GBS libraries. In brief, DNA normalized to $20 \mathrm{ng} / \mu \mathrm{L}$ was digested with Pst/and Mspl, barcoded and ligated to a common Y-adaptor using T4 DNA ligase. Then, all DNA samples were pooled, purified and PCR-amplified. DNA fragments of 200-300 bp were size-selected for sequencing on an Ion Proton sequencer (Thermo Fisher Scientific, Waltham, MA, USA). SNPs were called using a universal network-enabled analysis kit (UNEAK) and the reference-based TASSEL GBS pipeline (Elshire et al., 2011; Glaubitz et al., 2014). SNP positions were determined by mapping sequence reads to the barley reference genome Hordeum vulgare 11 (Mascher et al. 2017), and SNPs with minor allele frequency greater than $20 \%$, heterozygote frequency less than $5 \%$ and missing datapoints less than 11 were selected to map the target gene.

\section{Allelism test}

The greenbug resistance gene $R s g 2$ has not been mapped. Therefore, an $\mathrm{F}_{2}$ population was developed from the cross STARS1501B $\times$ WBDC053 to determine whether the greenbug resistance gene in WBDC053 is allelic to Rsg2. The $\mathrm{F}_{2}$ population was evaluated for response to greenbug biotype $\mathrm{E}$ in two replicates, using the protocols described above for the RIL population. 


\section{Mapping the greenbug resistance gene in WBDC053}

The ICI QTL Mapping V4.1 software program (Meng et al. 2015) was employed to map the greenbug resistance gene in WBDC053 using the Kosambi mapping function to convert recombination frequency into genetic distance (Kosambi 1944). The GBS-SNPs were grouped using a logarithm of the odds (LOD) threshold of 4.5, and ordered in each linkage group using the Recombination Counting and ORDering (RECORD) algorithm. The linkage map was fine-tuned using the sum of adjacent recombination fractions (SARF) at a window size of 7 as rippling criteria. MapChart 2.2 (Voorrips 2002) was used to draw the linkage map.

\section{Development of KASP markers}

Primer 3 (v. 4.0) (http://bioinfo.ut.ee/primer3-0.4.0/) was used to design primers. KASP assays were performed in an ABI ViiA 7 real-time PCR system (ThermoFisher Scientific, MA, USA), with $10 \mu \mathrm{L}$ reaction mixtures containing $5 \mu \mathrm{L} \mathrm{KASP}$ master mix, $12 \mu \mathrm{M}$ of each allele-specific primer, $30 \mu \mathrm{M}$ of common primer and $20 \mathrm{ng}$ genomic DNA. The PCR began at $30^{\circ} \mathrm{C}$ for $1 \mathrm{~min}$ and $94^{\circ} \mathrm{C}$ for $15 \mathrm{~min}$, and then continued for 40 cycles of $94^{\circ} \mathrm{C}$ for $20 \mathrm{~s}$ and $60^{\circ} \mathrm{C}$ for $1 \mathrm{~min}$, with a final step of $30^{\circ} \mathrm{C}$ for $1 \mathrm{~min}$. KASP marker data were analyzed using the ABI ViiA 7 software.

\section{Results}

\section{Responses of Weskan $\times$ WBDC053 RILs to greenbug biotype $\mathrm{E}$}

The two parents and their RILs were evaluated for responses to greenbug biotype E. WBDC053 was highly resistant, while Weskan was highly susceptible, resulting in the death of all plants at the end of the experiment. Of the 154 RILs, 84 were resistant and 64 were susceptible, which fit a 1:1 segregation ratio for a single gene $\left(\square^{2}=2.7, d f=1, p>0.1\right)$. The remaining six lines were still segregating, with an overall combined total of 99 resistant and 42 susceptible plants. Therefore, the greenbug resistance gene in WBDC053 is dominant $\left(\square^{2}=1.7, d f=1, p>0.18\right.$ for a $3: 1$ segregation ratio).

\section{Linkage analysis}

A total of 181.7 million high quality reads were generated from sequencing of the RIL population, with each RIL having 422,433-4,871,356 reads and 100.54 Mb-1.16 Gb sequence. These sequences were mapped to the barley reference sequence Hordeum vulgare $r$, which led to the identification of 69,506 SNPs with a minor allele frequency greater than 0.2. We further selected 3,347 SNPs which have no more than 10 missing datapoints in the RIL population and mapped 466, 521, 506, 238, 617, 402, and 540 GBSSNPs on chromosomes $\mathrm{H} 1$ to $\mathrm{H} 7$, respectively. The remaining 57 SNPs were not located to a specific chromosome. On average, each SNP had 2.5 missing datapoints in the RIL population.

Linkage analysis positioned the greenbug resistance gene in WBDC053, designated RsgWBDC053, to the distal end $(0-2.35 \mathrm{Mb})$ on the short arm of chromosome $2 \mathrm{H}$. The gene co-segregated with GBS-SNPs 
2H_1318811,2H_1368709 and 2H_1839499, and was $0.68 \mathrm{cM}$ distal to SNP 2H_2354645 in the map (Fig. 1).

\section{Allelism test}

Given that the genomic location of $R s g 2$ is still unknown, we performed an allelism test to determine whether RsgWBDC053s allelic to Rsg2 by evaluating an $\mathrm{F}_{2}$ population derived from STARS1501B $\times$ WBDC053. A total of $1,226 F_{2}$ plants, with 596 in replicate 1 and 630 in replicate 2 , were evaluated for response to greenbug biotype E. All 1,226 plants exhibited high resistance, indicating RsgWBDC366 is either allelic or very close to Rsg2.

\section{Development of KASP markers}

Two of the three GBS-SNPs co-segregating with RsgWBDC053,2H_1318811 and 2H_1839499, were converted to KASP markers KASP-Rsg053-1 and KASP-Rsg053-2, respectively. KASP-Rsg053-1 from $2 H_{-} 1318811$ was located at 1,318,811 bp in the Morex reference genome (Hordeum vulgare r1). WDBC053 carries the " $G$ " allele, while Weskan carries the "A" allele at this locus. The allele-specific primers locate from $1,318,791$ to $1,318,811 \mathrm{bp}$ and the common primer extends from 1,318,878 to 1,318,899 bp in the Hordeum vulgare 1 reference genome (Table 1). KASP-Rsg053-2 was converted from the GBS-SNP 2H_1839499 at 1,839,499 bp in the reference genome, where Weskan and WDBC053 carry the " $C$ " and " $T$ " alleles, respectively. We used the genomic sequence from 1,839,479 to $1,839,499$ bp to design the allele-specific primers and 1,839,544 to 1,939,563 bp to design the common primer (Table 1).

KASP-Rsg053-1 and KASP-Rsg053-2 were used to genotype the RIL population (Fig. 2), and the KASP genotyping results were in complete agreement with the GBS-SNP data, indicating that KASP-Rsg053-1 and $K A S P-R s g 053-2$ can be reliably used to tag $R s g 053$ in barley breeding. These markers can facilitate the introgression of the Rsg gene in WBDC053 into locally adapted barley breeding lines.

\section{Candidate genes in the target region}

The $R s g$ gene in WBDC053 resides in the interval of $0-2,354,645$ bp (SNP 2H_2354645) in the terminal region of chromosome $2 \mathrm{HS}$. There are 59 annotated genes in this region with 46 genes encoding proteins with known functions in the Hordeum vulgare 11 reference sequence (Table 2). Among the 46 genes, 15 are R genes that encode proteins containing leucine-rich-repeat (LRR), including HORVU2Hr1G000150, HORVU2Hr1G000160, HORVU2Hr1G000170, HORVU2Hr1G000180, HORVU2Hr1G000190, HORVU2Hr1G000200, HORVU2Hr1G000290, HORVU2Hr1G000510, HORVU2Hr1G000560, HORVU2Hr1G000630, HORVU2Hr1G000640, HORVU2Hr1G000700, HORVU2Hr1G000770, HORVU2Hr1G000780, and HORVU2Hr1G000900. Given that the LRR-containing proteins are usually associated with innate immune responses in plants, they are putative candidates for the $R s g$ gene in WBDC053. Genes encoding cytochrome P450-related proteins, aldo/keto reductase, protein phosphatase 2, L-3-cyanoalanine synthase/cysteine synthase, transferase, tetrahydroberberine oxidase/THB oxidase, 
and hexokinase etc. were also identified in this region (Table 2). High resolution mapping will be required to identify the candidate Rsg gene in WBDC053.

\section{Discussion}

\section{$R s g W B D C 053$ is a new gene for greenbug resistance}

Wild species has been proven to be an important source for greenbug resistance in small grain cereals. In wheat, seven of the eight greenbug resistance genes originated from wild species or wheat relatives, including Gb5 from Ae. speltoides, Gb2 and Gb6 from rye, and Gb3, Gb4, Gb7, and Gb8 from Ae. tauschii (Xu et al. 2020). In this study, we identified a greenbug resistance gene in wild barley for the first time. This finding highlights the value of this subspecies for identifying new resistance genes of value for barley (Henningsen et al. 2021).

The $R s g$ gene in WBDC053 was mapped on chromosome $2 \mathrm{H}$ in this study, and $R s g 1$ was previously mapped to chromosome $3 \mathrm{H}$, demonstrating they are different genes at two unique loci. The chromosome location of Rsg2 has not been reported to date; however, a previous study indicated that STARS 1051B (the source of $R s g 2$ ) and WDBC053 were resistant and susceptible to greenbug biotype F, respectively (Armstrong et al. 2015). This indicates that the two genes are indeed different from each other. To further resolve the relationship between the $R s g$ genes in the two resistance sources, we conducted an allelism test, and the results suggested the Rsg gene in WBDC053 either lies at a new locus closely linked with $R s g 2$ or is an allele at the Rsg2 locus. Given that a considerable number of $\mathrm{R}$ genes reside in the target region, the greenbug resistance gene in WBDC053 will be given the temporary designation of RsgWBDC053 until high resolution mapping studies are conducted.

Tocho et al. (2013) mapped a quantitative trait locus (QTL), QGb.un/p-2H, for reduced foliar area and dry weight by greenbug infestation on chromosome $2 \mathrm{H}$; thus, this genomic region might harbor a QTL for greenbug tolerance. However, Tocho et al. (2013) focused on tolerance and the two parents in that study showed slight difference in chlorosis. RsgWBDC053 likely not QGb.unlp-2H because WDBC053 shows high resistance to greenbug.

RsgWBDC053 was mapped to a $2.35 \mathrm{Mb}$ interval in the terminal region of chromosome $2 \mathrm{H}$. In this interval, 59 annotated genes were identified with 15 genes carrying LRR protein domains. LRR protein domains commonly confer plant disease resistance by recognizing products of avirulent genes in pathogens. Some of these genes are either very close to or between GBS-SNPs markers that cosegregated with greenbug resistance in the RIL population. For example, HORVU2Hr1G000510 is 1,579 bp distal to 2H_1318811, and HORVU2Hr1G000560, HORVU2Hr1G000630, HORVU2Hr1G000640, HORVU2Hr1G000700, HORVU2Hr1G000770, HORVU2Hr1G000780, and HORVU2Hr1G000900 reside between $2 H_{-} 1318811$ and $2 H_{-}$1839499. To date, no aphid resistance gene has been cloned and the mechanisms underlying greenbug or any other aphid resistance are still elusive. Given that LRR genes often provide plant innate immune responses, these LRR genes are good candidates for RsgWBDC053. Further fine-mapping of RsgWBDCO53 is needed to identify candidate genes and also resolve its 
relationship to $R s g 2$. This should be feasible given the availability of residual heterozygosity in the RIL population. Of the $154 \mathrm{~F}_{6}$ RILs evaluated in this study, six lines were segregating for resistance to greenbug biotype $\mathrm{E}$. The corresponding $\mathrm{F}_{5}$ lines of these RILs can be advanced for fine mapping RsgWBDC053. Since the gene resides in the gene-rich terminal end of chromosome $3 \mathrm{H}$, we expect high recombination frequency in this region, which may lead to the identification of the causal variant. Additionally, WBDC053 and other WBDC accessions are currently being sequenced by the International Wild Barley Sequencing Consortium (https://iwbsc.umn.edu/). This effort will facilitate the cloning of the greenbug resistance gene from this wild barley accession.

\section{Use of RsgWBDCO53 in barley breeding}

Since the greenbug resistance gene identified in this study was from wild barley, it is likely that some undesirable genes will be carried along when introgressing it into adapted germplasm. Thus, pre-breeding will be necessary to efficiently transfer RsgWBDC053 into modern barley cultivars. The KASP markers developed in this study co-segregated with RsgWBDC053 in the RIL population and can be used for marker-assisted selection in barley breeding. WBDC053 is resistant to greenbug biotypes B, C, E, I, TX1, WY4, WY81, WY12 MC, and WY86 (Armstrong et al. 2016). Given that Rsg1 is overcome by biotypes TX1 and WY81 (Armstrong et al. 2016), the resistance gene in WBDC053 will be valuable for enhancing greenbug resistance in combination with $R s g 1$. Molecular markers flanking $R s g 1$ are available (Azhaguvel et al. 2014). Further conversion of those markers into KASP markers is still necessary to meet the requirements for high throughput screening of breeding materials. Moreover, $R s g 2$ has been recently transferred to elite barley lines by recurrent backcrossing of STARS1501B, a selection from PI 426756, to Weskan, leading to the development of STARS1502B. STARS1502B showed similar grain yield, plant height, and test weight in fields as Weskan (Mornhinweg et al. 2018), suggesting that Rsg2 does not negatively affect yield or quality traits. Rsg2 is either allelic or very close to RsgWBDC053. Thus, the KASP markers developed in this study have the potential to tag Rsg 2 in barley breeding, depending on whether they are polymorphic between the parents of breeding populations.

The only WBDC accessions found resistant to greenbug biotype by Armstrong et al. (2016) were WBDC053 from Pakistan, WBDC157 from Iraq, and WBDC177 and WBDC366 from Turkmenistan. This suggests that greenbug resistance may have originated in wild barley populations of South and Central Asia and Middle East. To identify additional sources of resistance, greater sampling and testing of wild barley accessions from this region should be conducted. In addition to greenbug resistance, WBDC053 possesses a high level of resistance to powdery mildew, an important foliar disease of barley caused by the fungus Blumeria graminis f. sp. hordei. The resistance spectrum of WBDC053 was also very wide as it exhibited low infection types to 38 of 40 B. graminis f. sp. hordei pathotypes tested (Ames et al. 2015).

\section{Declarations}

\section{Acknowledgements}


Mention of trade names or commercial products in this publication is solely for the purpose of providing specific information and does not imply recommendation or endorsement by the USDA. The USDA is an equal opportunity provider and employer.

\section{Conflict of interest}

On behalf of all authors, the corresponding authors state that there is no conflict of interest.

\section{Author contributions}

$X X, D M$ and GB designed experiments; $D M, G L, A B$ and $B L$ conducted genotyping and phenotyping experiments; BJS provided genetic materials; $X X$ drafted the manuscript and all authors revised the manuscript and approved the final version of the manuscript.

\section{References}

Ames N, Dreiseitl A, Steffenson BJ, Muehlbauer GJ (2015) Mining wild barley for powdery mildew resistance. Plant Pathol 64:1396-406

Armstrong JS, Mornhinweg DW, Payton ME, Puterka GJ (2015) The discovery of resistant sources of spring barley, Hordeum vulgare ssp. spontaneum, and unique greenbug biotypes. J Econ Entomol 109:434-438

Azhaguvel P, Mornhinweg D, Vidya-Saraswathi D, Rudd JC, Chekhovskiy K, Saha M, Close TJ, Dahleen LS, Weng Y (2014) Molecular mapping of greenbug (Schizaphis graminum) resistance gene Rsg1 in barley. Plant Breed 133:227-233

Edwards LH, Smith EL, Pass H, Morgan GH (1985) Registration of Post barley. Crop Sci 25:363

Elshire RJ, Glaubitz JC, Sun Q, Poland JA, Kawamoto K, Buckler ES, Mitchell SE (2011) A robust, simple genotyping-by-sequencing (GBS) approach for high diversity species. PloS ONE. 6:e19379.

FAOSTAT (2017) Crops/Regions/World List/Production Quantity for Barley, 2017

Dubcovsky J, Galvez AF, Dvořák J (1994) Comparison of the geneticorganization of the early salt-stressresponse gene system in salt tolerant Lophopyrum elongatum and salt-sensitive wheat. Theor Appl Genet 87:957-964

Giles KL, Royer TA, Elliott NC, Kindler SD (2000) Development and validation of a binomial sequential sampling plan for greenbug (Homoptera: Aphididae) infesting winter wheat in the southern plains. J Econ Entomol 93:1522-1530

Giles KL, Jones DB, Royer TA, Elliott NC, Kindler SD (2003) Development of a sampling plan in winter wheat that estimates cereal aphid parasitism levels and predicts population suppression. J Econ Entomol 
Glaubitz JC, Casstevens TM, Lu F, Harriman J, Elshire RJ, Sun Q, Buckler ES (2014) TASSEL-GBS: a high capacity genotyping by sequencing analysis pipeline. PloS ONE 9:e90346.

Kosambi DD (1944) The estimation of map distances from recombination values. Ann Eugen 12:172175

Mascher M, Wu S, Amand PS, Stein N, Poland J (2013) Application of genotyping-by-sequencing on semiconductor sequencing platforms: a comparison of genetic and reference-based marker ordering in barley. PloS ONE 8: e76925

Mascher M, Gundlach H, Himmelbach A, Beier S, Twardziok SO, Wicker T, Radchuk V, Dockter C, Hedley $\mathrm{PE}$, Russell J, Bayer M (2017) A chromosome conformation capture ordered sequence of the barley genome. Nature 544:427-433

Meng L, Li H, Zhang L, Wang J (2015) QTL IciMapping: integrated software for genetic linkage map construction and quantitative trait locus mapping in biparental populations. Crop J 3:269-283

Mornhinweg DW, Edwards LH, Smith EL, Morgan GH, Webster JA, Porter DR, Carver BF (2004) Registration of 'Post 90' barley. Crop Sci 44:2263-2264

Mornhinweg DW, Armstrong JS, Carver BF, Springer TL (2018) Registration of STARS 1501B and STARS 1502B barley germplasm with $R s g 2$ resistance to greenbug. J Plant Regist 12:237-240

Porter DR, Burd JD, Mornhinweg DW (2007) Differentiating greenbug resistance genes in barley. Euphytica 153:11-14

Puterka GJ, Peters DC, Kerns DL, Slosser JE, Bush L, Worrall DW, McNew RW (1988) Designation of two new greenbug (Homoptera: Aphididae) biotypes G and H. J Econ Entomol 81:1754-1759.

Starks KJ, Burton RL (1977) Preventing greenbug outbreaks. Report no. 309, USDASci. Educ. Admin. Leaflet. Washington, DC

Steffenson BJ, Olivera P, Roy JK, Jin Y, Smith KP, Muehlbauer GJ (2007) A walk on the wild side: mining wild wheat and barley collections for rust resistance genes. Aust J Agric Res 58:532-544

Tocho E, Börner A, Lohwasser U, Castro AM (2013) Mapping and candidate gene identification of loci determining tolerance to greenbug (Schizaphis graminum, Rondani) in barley. Euphytica 191:173-182

Ullrich, S. E. (2011). Barley: Production, Improvement and Uses. Wiley-Blackwell. Ames, IA, USA.

Voorrips RE (2002) MapChart: software for the graphical presentation of linkage maps and QTLs. J Hered 93:77-78 
Webster JA, Starks KJ (1984) Sources of resistance in barley to two biotypes of the greenbug Schizaphis graminum (Rondani), Homoptera: Aphididae. Protect Ecol 6:51-55

Xu X, Li G, Carver BF, Armstrong JS (2020) Gb8, a new gene conferring resistance to economically important greenbug biotypes in wheat. Theor Appl Genet 133:615-622

\section{Tables}

Table 1. Alleles, allele-specific primers, and Common primers of KASP-Rsg053-1 and KASP-Rsg053-2

\begin{tabular}{|llll|}
\hline Marker & Alleles* & Allele-specific Primer & Common Primer \\
\hline KASP-Rsg053-1 & G/A & CCAGGTCCCTGCAGTTGAAG & ACTACTGGCTGGTGAATTCCG \\
& & CCAGGTCCCTGCAGTTGAAA & \\
KASP-Rsg053-2 & T/C & CTGCTTGGAACCATGACACC & GTTCATGTGCATAGCGCCC \\
& & CTGCTTGGAACCATGACACT & \\
\hline
\end{tabular}

*: WBDC053/Weskan

Table 2 Genomic locations and function annotation of the candidate genes in the terminal region of chromosome 2H where RsgWBDC053 was located. 


\begin{tabular}{|c|c|c|}
\hline Gene & $\begin{array}{l}\text { Genomic location } \\
\text { (bp) }\end{array}$ & Function \\
\hline HORVU2Hr1G000010 & 24939..32359 & Polycomb group protein \\
\hline HORVU2Hr1G000050 & 139300..149044 & ALDO/KETO reductase \\
\hline HORVU2Hr1G000060 & 182842..185828 & Ethylene response sensor-2-related \\
\hline HORVU2Hr1G000140 & $303954 . .304483$ & ALDO/KETO reductase \\
\hline HORVU2Hr1G000090 & 207003..213166 & Protein phosphatase $2 \mathrm{C}$ (PP2C) \\
\hline HORVU2Hr1G000150 & $555110 . .557071$ & Leucine-rich repeat containing protein \\
\hline HORVU2Hr1G000160 & $574243 . .576804$ & Leucine-rich repeat containing protein \\
\hline HORVU2Hr1G000170 & $607918 . .609884$ & Leucine-rich repeat containing protein \\
\hline HORVU2Hr1G000180 & $619057 . .624665$ & Leucine-rich repeat containing protein \\
\hline HORVU2Hr1G000200 & $645032 . .647593$ & Leucine-rich repeat containing protein \\
\hline HORVU2Hr1G000190 & $693030 . .712161$ & Leucine-rich repeat containing protein \\
\hline HORVU2Hr1G000250 & 723583..723698 & $\begin{array}{l}\text { Bifunctional L-3-cyanoalanine synthase/cysteine } \\
\text { synthase/cysteine synthase C1, mitochondrial }\end{array}$ \\
\hline HORVU2Hr1G000260 & $761023 . .766626$ & $\begin{array}{l}\text { Bifunctional L-3-cyanoalanine synthase/cysteine } \\
\text { synthase/cysteine synthase C1, mitochondrial }\end{array}$ \\
\hline HORVU2Hr1G000270 & 764403..765912 & Predicted membrane protein \\
\hline HORVU2Hr1G000280 & 766431..831248 & $\begin{array}{l}\text { L-3-cyanoalanine synthase/ cysteine synthase } \\
\text { (ATCYSC1) }\end{array}$ \\
\hline HORVU2Hr1G000290 & 783497..792896 & Leucine-rich repeat containing protein \\
\hline HORVU2Hr1G000360 & $923158 . .929823$ & Sequence-specific DNA binding transportation factor \\
\hline HORVU2Hr1G000380 & $952979 . .954487$ & C2H2-type zinc finger (zf-C2H2_6) \\
\hline HORVU2Hr1G000390 & 1021350..1032930 & Polycomb protein Suz12 \\
\hline HORVU2Hr1G000410 & 1146999..1148640 & Transferase family (Transferase) \\
\hline HORVU2Hr1G000420 & 1183898..1186327 & Transferase family (Transferase) \\
\hline HORVU2Hr1G000430 & 1196655..1203088 & Cytochrome P450 CYP4/CYP19/CYP26 subfamilies \\
\hline HORVU2Hr1G000480 & 1235192..1236988 & Cytochrome P450 CYP4/CYP19/CYP26 subfamilies \\
\hline HORVU2Hr1G000490 & 1265575..1266439 & Tetrahydroberberine oxidase / THB oxidas \\
\hline HORVU2Hr1G000510 & 1308095..1317214 & Leucine-rich repeat containing protein \\
\hline HORVU2Hr1G000530 & 1317594..1320213 & Tetrahydroberberine oxidase / THB oxidase \\
\hline
\end{tabular}




\begin{tabular}{|llll|}
\hline HORVU2Hr1G000540 & $1322179 . .1323992$ & Tetrahydroberberine oxidase / THB oxidase \\
\hline HORVU2Hr1G000550 & $1326577 . .1327546$ & Cannabidiolic acid synthase / CBDA synthase \\
\hline HORVU2Hr1G000560 & $1361514 . .1366863$ & Leucine-rich repeat containing protein \\
\hline HORVU2Hr1G000590 & $1380974 . .1386210$ & G-patch domain (G-patch) \\
\hline HORVU2Hr1G000630 & $1465139 . .1466444$ & Leucine-rich repeat containing protein \\
\hline HORVU2Hr1G000640 & $1466498 . .1468456$ & Leucine-rich repeat containing protein \\
\hline HORVU2Hr1G000660 & $1491544 . .1493077$ & Cytochrome P450 CYP4/CYP19/CYP26 subfamilie \\
\hline HORVU2Hr1G000670 & $1501961 . .1503257$ & Cytochrome P450 81D1-related \\
\hline HORVU2Hr1G000700 & $1539568 . .1543563$ & Leucine-rich repeat containing protein \\
\hline HORVU2Hr1G000770 & $1712040 . .1719477$ & Leucine-rich repeat containing protein \\
\hline HORVU2Hr1G000780 & $1681391 . .1683451$ & Leucine-rich repeat containing protein \\
\hline HORVU2Hr1G000840 & $1729315 . .1730862$ & Methanol O-anthraniloyltransferase \\
\hline HORVU2Hr1G000890 & $1766506 . .1768035$ & Cytochrome P450 CYP2 subfamil \\
\hline HORVU2Hr1G000900 & $1797984 . .1801262$ & Leucine-rich repeat containing protein \\
\hline HORVU2Hr1G000940 & $2084734 . .2097842$ & $\begin{array}{l}\text { Histone-lysine N-methyltransferase, H3 lysine-36 } \\
\text { specifi }\end{array}$ \\
\hline HORVU2Hr1G000990 & $2145801 . .2162290$ & Hexokinase (HK) \\
\hline HORVU2Hr1G001070 & $2245699 . .2246897$ & Late embryogenesis abundant protein (LEA_2) \\
\hline HORVU2Hr1G001110 & $2335789 . .2337923$ & Cytochrome P450 76C1 \\
\hline HORVU2Hr1G001120 & $2347405 . .2351445$ & $\begin{array}{l}\text { Terpene synthase, N-terminal domain (Terpene_synth) } \\
\text { // Terpene synthase family, metal binding domain }\end{array}$ \\
\hline & & (Terpene_synth_C) \\
\hline
\end{tabular}

\section{Figures}




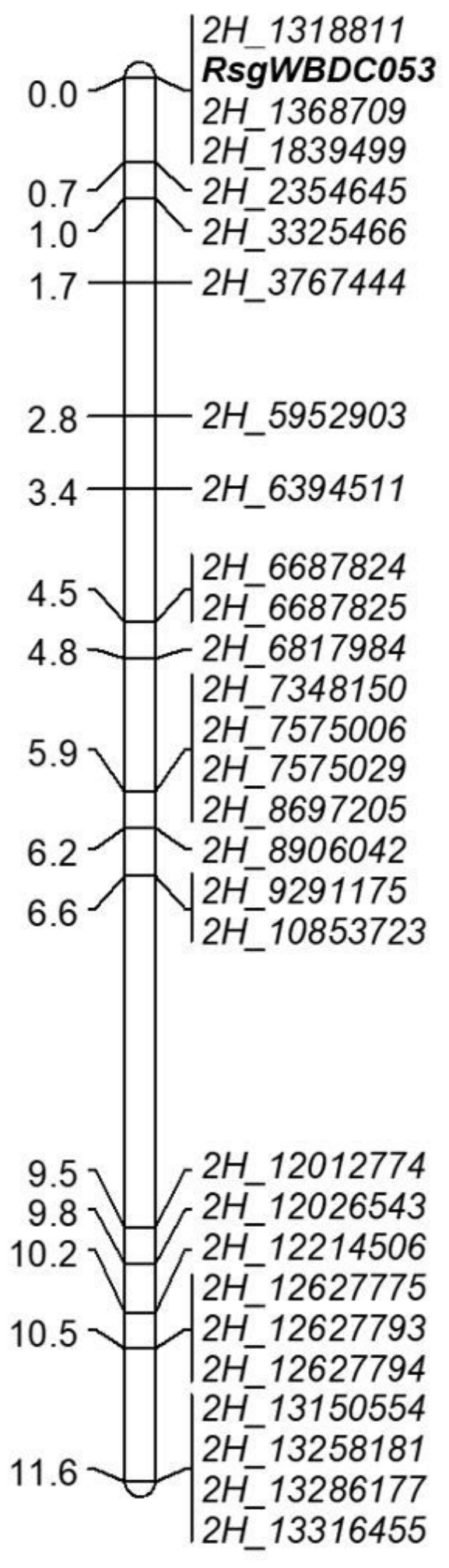

\section{Figure 1}

A linkage map containing RsgWBDC053 in the terminal region of chromosome $2 \mathrm{H}$. The GBS-SNPs and genetic distances (in $\mathrm{CM}$ ) are shown on the right and left sides of the linkage map, respectively. Each SNP was named using a combination of its chromosome and physical location in the Hordeum vulgare r1 reference sequence (in bp). Only a portion of the chromosome $2 \mathrm{H}$ linkage map is shown. 

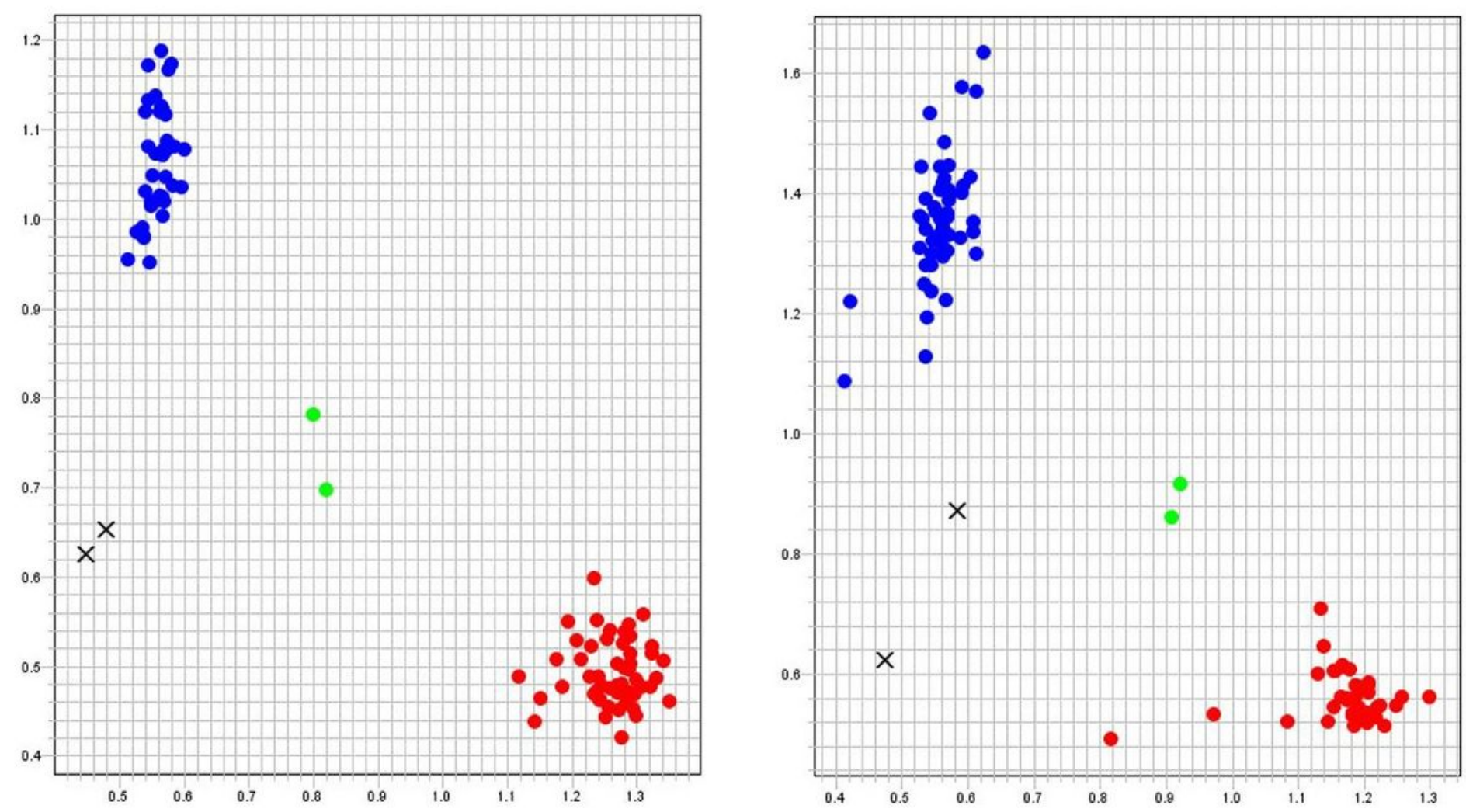

Figure 2

Segregation of the WBDC053 (red dots), Weskan (blue dots) and heterozygous (green dots) alleles at the KASP-Rsg053-1 (left) and KASP-Rsg053-2 (right) loci in a RIL population from WBDC053 $\times$ Weskan. The " $x$ " represents negative control. 